\title{
Traumatic Spinal Cord Injury in Nepal
}

\section{Shrestha D}

Spinal cord injury is devastating injury, which has serious impact not only to the individual but also to family members, society and the nations as a whole because the available treatment options are expensive, prolong, resource consuming and do not always fulfill the expectation of patient and family members. Worldwide incidence of spinal cord injury has been reported up to 236-1009/million/year. ${ }^{1}$ The incidence, etiology and gender distribution varies significantly in different geographical areas of the world. In Asia, incidence of spinal cord injury is $12.06-61.6 /$ million/year. $^{2}$ Incidence of spinal cord injury has not been reported in Nepal. However, two studies from eastern Nepal have reported 382 spinal injuries (149 cervical in between 2001 to 2004 and 233 spinal cord injury in between 1997 to 2001). ${ }^{3,4}$

Over last few years, numbers of spinal cord injury patients are increasing in Nepal. Major etiology of spinal injury in Nepal is related with fall related injury unlike in western countries where Motor Vehicle Accident (MVA) tops the list. With the increasing number of road traffic accidents, MVA has been projected as major etiology of spinal cord injury in Nepal and other developing countries. ${ }^{5}$ MVA contributes $59.5 \%$ of spinal cord injury in other Asian countries. ${ }^{2}$ Gender wise, males are predominantly involved and most of them are in productive age group with range 26 to $56 \mathrm{yrs}^{2}{ }^{2}$ Injury prevention and emergency management is another challenge for Nepal to prevent spinal cord injury. Some of the injuries were preventable with simple precaution like using safety measure at the work place. Trauma patients often reach to the hospital without neck or spine immobilization. Study from eastern Nepal revealed that $81 \%$ of cervical spinal cord injury patients were brought to hospital without neck immobilization. ${ }^{3}$ Awareness among ambulance drivers or even lay persons about need of immobilization of neck and back during transportation of patients can prevent many catastrophes.

Management of spinal cord injury is extremely difficult in the resource-limited countries like Nepal. Besides economic constraints, limiting factors for the optimal management of these patients are lack of trained personals and the well-equipped center and awareness among patients and family members. In 2014, Ministry of Health and Population (MoHP) announced financial support of NPR one lakh to the patient of spinal cord injury patient. But this fund is available through only few designated hospital in Kathmandu and hence the patients with spinal cord injury need to travel to capital to be benefitted with financial help from the government. Many patients, hospitals and even health care personnel may not be aware of government financial support to the spinal cord injury patients.

With increasing interest and development of surgical skills among orthoapedic/neurosuregeon, and the hospital equipped with intensive care unit, operative treatment options for spinal cord injury patients are available in major cities in Nepal and as result, patients do not need to suffer from complications related with prolonged immobilization, such as; pressure ulcers, hypostatic pneumonia, urinary tract infection etc. But in the absence of standard treatment protocols or inadequate training or less than ideal environment for surgical treatment, many operated patients are not getting benefit as expected or sometimes suffering from complications related to surgery. Sometimes patients are not adequately counseled about what to expect and what not after surgery that makes post-operative rehabilitation extremely difficult. To provide optimal and ideal care, both operative and non-operative to the patient with acute spinal cord injury, there is a need of center or hospital or at least a unit where patients can be comprehensively treated with holistic approach by a spine surgeon or trained orthopaedic/neurosurgeon so that patients get treatment based upon evidence rather than assumption. This is usual practice in most the developed countries where few hospitals/centers/ units are designated as a referral center for specific and complicated problems like spinal cord injury.

Rehabilitation of spinal cord injury patients whether treated with or without surgeries are most of the time overlooked. Treating surgeons usually consider that his/her role is completed once the patient is discharged from the hospital. In the absence of proper rehabilitation, spinal cord injury patients are more vulnerable to suffer from secondary health complications ranging from pressure ulcers to death. First year survival after traumatic cervical spinal cord injury in developing country is extremely poor. There are very few rehabilitation centers, run by nongovernmental organization such as Spinal Cord Injury Rehabilitation Center in Kavre and Green Pasture Rehabilitation Center in Pokhara for spinal cord injury patients. Patients usually do not get benefit from financial support provided by the government during rehabilitation phase because the support is usually spent for acute management in the hospital rather than in rehabilitation. Without physical, 
occupational and psychological rehabilitation, integration of spinal cord injury patients in family and community is extremely difficult and hence they are neglected. But with comprehensive training by the experts and some small modifications in home and working places, they can be independent and live normal productive life.

To provide ideal and optimal care (both acute and rehabilitation) to spinal cord injury patients as well as to prevent such injuries, therefore, a policy should be prepared with active involvement of MoHP and other stakeholders and should clearly outline about required training and skill of treating surgeons, hospitals, rehabilitation centers, supportive facilities and system of referral whenever necessary. Financial support by government should be available for other hospital in the country wherever services to spinal cord injury is provided and extended to rehabilitation purpose as well.

(Author is affiliated to the Department of Orthopedics and Traumatology, Dhulikhel Hospital, Kathmandu University Hospital)

\section{REFERENCES}

1. Cripps RA, Lee BB, Wing P, Weerts E, Mackay J, Brown D. A global map for traumatic spinal cord injury epidemiology: towards a living data repository for injury prevention. Spinal Cord 2011 Apr;49(4):493-501.

2. Burns SA, O'Connell C. The challenge of spinal cord injury care in the developing world. The Journal of Spinal Cord Medicine. $2012 ; 35$ (1): 3-8.

3. Shrestha D, Garg M, Singh GK, Singh MP, Sharma UK. Cervical spine injuries in a teaching hospital of eastern region of Nepal: a clinico-epidemiological study. J Nepal Med Assoc 2007; 46: 107-11.

4. Lakhey S, Jha N, Shrestha BP, Niraula S. Aetioepidemiological profile of spinal injury in eastern Nepal. Trop Doct 2005; 35: 231-3.

5. World Health Organization. World report on road traffic injury prevention. Geneva Switzerland: WHO Publications 2004. 